\title{
Hands-On Tutorial
}

\section{Coarse-Grained Reconfigurable Architectures - Compilation and Exploration}

\author{
Tom Vander Aa \\ imec \\ Kapeldreef 75 , \\ Leuven, Belgium \\ vanderaa@imec.be
}

\author{
Panagiotis Theocharis \\ Ghent University \\ Sint Pietersnieuwstraat 41, \\ Ghent, Belgium \\ Panagiotis.Theocharis@elis.ugent.be
}

\begin{abstract}
CGRAs consist of an array of a large number of functional units (FUs) interconnected by a mesh style network. Register files are distributed throughout the CGRAs to hold temporary values and are accessible only by a subset of FUs. The FUs can execute common word-level operations, including addition, subtraction, and multiplication. CGRA processors accelerate inner loops of applications by exploiting instruction level parallelism (ILP) and in some cases also data-level and task-level parallelism (DLP \& TLP).

The aim of this tutorial is to give insight in CGRA architectures, their compilation techniques, and to experience first hand how to do source code mapping on a CGRA. Therefore the tutorial consists of presentations as well as a hands-on session.
\end{abstract}

\section{Categories and Subject Descriptors}

C.1.4 [Computer Systems Organization]: Processor

Architectures - parallel architectures.

General Terms: Performance, Design.

Keywords: CGRA, reconfigurable architecture, compiler, source code mapping.

\section{SPEAKERS' BIOGRAPHY}

Tom Vander $\mathrm{Aa}$ is a senior researcher in the Wireless Communication group of IMEC working on low energy high performance architectures and compilation techniques.
Since 2005 he has been working on the ADRES coarse-grain array processor. In 2005, Tom Vander Aa obtained a $\mathrm{PhD}$ in electrical engineering from KULeuven, Leuven, Belgium on energy optimization for instruction memory of embedded processors. He has a master in computer science degree, also from KULeuven and is a member of the IEEE.

Panagiotis Theocharis is a doctoral researcher in the Computer Systems Lab of Ghent University working on split compilation for accelerator-based multicores. He holds a diploma in electrical and computer engineering from the National Technical University of Athens and is a member of the ACM and the IEEE.

\section{TOPICS}

Below is a list of topics covered during the tutorial:

- Presentation: Introduction to CGRAs and their compilation techniques, taking the ADRES CGRA as an example (45')

- Presentation + demo: Introduction to the DRESC tool chain (15')

- Guided hands-on: The audience can have a first try of compilation and simulation. (30')

- Presentation: How to optimize your source code for CGRAs (30')

- Hands-on: The audience will try to optimize a prepared example for the CGRA. (30')

- Presentation: Current research in CGRA compilation: building optimizing compilers using machine learning. (30')

Copyright is held by the author/owner(s).

CODES+ISSS'12, October 7-12, 2012, Tampere, Finland.

ACM 978-1-4503-1426-8/12/09. 